\title{
The Realisitic Value of Frozen Section in Intraoperative Decision Making In Malignancies - in The Public Sector in India
}

\author{
Dr. Kavitha Sukumar MS., $\mathrm{MCh}^{1}$,Dr. Sivakumari Sivarajan,MS.,MCh ${ }^{2}$ \\ ${ }^{1}$ Assistant Professor Of Surgical Oncology,Tamilnadu Government Multi Super Speciality Hospital , \\ Omandurar, TN Dr. MGR Medical University, Chennai, Tamilnadu, India. \\ ${ }^{2}$ Assistant Professor Of Surgical Oncology,Tamilnadu Government Multi Super Speciality Hospital, \\ Omandurar, TN Dr. MGR Medical University, Chennai, Tamilnadu, India.
}

\begin{abstract}
Frozen Section is an important tool in the armamentarium of the surgical oncologist. The value of frozen section in oncology is established well .However, in India, especially in the Public sector, it is available in very few centers. . Thus, this study aims to determine the realistic value of Frozen section in malignancies in the public sector.Frozen section of 179 samples from 120 cases were analysed. The most common indication for intraoperative evaluation was to assess suspicious nodes, accounting for $44.13 \%$. Head and neck and gynecologic tumors were the most common indications for study, accounting for $57 \%$ of cases. $16 / 179$ samples were deferred for final histopathological analysis, giving a deferral rate of 8.9\%. On comparison with paraffin block histopathology, the concordance and discordant rates of frozen section were $90.72 \%$ and $10.42 \%$ respectively. The sensitivity, specificity, positive predictive value and negative predictive value of frozen section were $80 \%, 95.2 \%, 90.56 \%$ and $89.2 \%$ respectively. Thus, it can be concluded that Frozen section is reliable and accurate for intraoperative evaluation of nodes, margin status and for the primary diagnosis of most tumors and it is feasible to run a reasonably accurate frozen section facility at the public sector.
\end{abstract}

Keywords: Frozen section, Intraoperative diagnosis, Malignancy

\section{Introduction}

The Frozen section is a laboratory procedure to perform rapid microscopic pathological analysis of specimen. It is an important tool in the armamentarium of the surgical oncologist for intraoperative diagnosis, which provides direct evidence and foundation for the extent of the surgery. Frozen section has been widely studied in the west and its role in oncology is well established. However, in India, especially in the Public sector, Frozen section is available in very few centers. Equipment cost, maintenance, availability of technicians and trained pathologists are all hurdles in sustaining the frozen section facility. Thus, this study aims to determine the realistic value of Frozen section in malignancies in the public sector.

\section{Aim of Study}

To determine the efficacy of frozen section in the intraoperative management of various malignancies, by comparing it with the permanent paraffin section, in the setting of a Public sector in India

\section{Materials And Methods}

The study was a prospective study conducted at the department of Surgical Oncology, Government Royapetah hospital, Kilpauk Medical College, Chennai between October 2011 and January 2014. The inclusion criteria were

3.1 : 1Patients requiring frozen section for primary diagnosis, when preoperative biopsy not possible and frozen section would change the extent of surgery

3.2: In patients with known malignancy, to assess margin status or to assess the extent of disease

3.3: For assessment of lymph nodes, For establishing a diagnosis or to assess involvement of the node, in case of known primary, Sentinel node evaluation in selected cases

3.4: Assessment of specimen adequacy, in difficult biopsies

Cases in which intraoperative pathological diagnosis has no immediate surgical implications, heavily ossified tissue or when lesion size less than $1 \mathrm{~cm}$ with no preoperative diagnosis, were excluded from the study.

Frozen section analysis of 120 patients were performed in the study period. The clinical details of the patients were collected preoperatively and informed consent for frozen section analysis was obtained. The patients were informed of the various management options and the change in the intraoperative management as per the frozen section reports. In the laboratory, the specimen was initially grossed by the pathologist, the most representative area selected and sampling was done. The sample was then embedded in a gel like medium consisting of polyethylene glycol and polyvinyl alcohol and frozen rapidly in a cryostat machine to - 20 to $-30^{\circ}$ 
Celsius. The Cryostat used in our hospital was Leica CM $1510 \mathrm{~S}$. After adequate freezing, the sample was sectioned with the microtome portion of the cryostat . 5 micron section thickness was most commonly used. The section was picked up on a glass slide using the brush technique, fixed with $95 \%$ ethanol and stained with hematoxylin \& eosin. The slides were interpreted by the pathologist. Time taken for reporting was documented. With the frozen section report, the extent of surgery was confirmed and surgery was completed. The surgical specimen were sent for permanent paraffin section. A final analysis was done and the frozen section was compared with the final histopathology report as to whether the frozen section was concordant / discordant / indeterminate.

The sensitivity, specificity, positive and negative predictive value of frozen section were calculated. The accuracy rate, discordance rate and deferral rate of frozen section were calculated and compared with various studies .

Concordance was defined as an adequate intraoperative frozen section evaluation which had complete diagnostic agreement with the final histopathology examination.

Discordance was defined as an adequate frozen section evaluation with diagnostic disagreement with final histopathology.

The number of cases in which diagnosis were deferred were analyzed and excluded from the calculations of concordant and discordant rates.

\section{Results}

A total of 179 samples from 120 cases were analyzed. The most common indication for intraoperative evaluation was to assess suspicious nodes, accounting for 44.13\%[ 79/179], followed by frozen section of the tumor for primary diagnosis which accounted for $32.40 \%$. [ 58/179 ]. The other indications were to assess margins , sentinel nodes and metastatic tumor deposits at $10.61 \%, 7.26 \%$ and $5.58 \%$ respectively.

Head and neck and gynecologic tumors were the most common indications for frozen section, accounting for $57 \%$ of cases. This reflects the case distribution in our centre where head and neck and gynecologic tumors are the major contributors. Among head and neck malignancies, squamous cell carcinomas accounted for the majority[ $75 \%$ ] of requests, again reflecting on the case distribution in our centre. Among gynaecological tumors, adnexal masses were most commonly sought for frozen section analysis. This can be explained by the fact the preoperative diagnosis is often not available for ovarian masses which necessitates intraoperative evaluation in many of these cases.

Preoperative diagnosis was not available in $49 / 120$ patients. Adnexal masses were the most common specimens sent for primary diagnosis, since preoperative tissue biopsy is contraindicated in operable cases. Retroperitoneal masses, small intestinal masses, pancreatic masses were also subject to primary diagnosis. This is due to the inefficiency of the available diagnostic modalities for preoperative diagnosis. In addition, some breast, bone and soft tissue tumors was sent for primary diagnosis. These cases were those with extensive necrosis and multiple preoperative core needle biopsies were reported negative for malignancy

The samples sent were adequate for frozen section evaluation in $92.17 \%$. However they were inadequate for evaluation in $7.8 \%$. The reasons for inadequacy were

4.1: Tissue too tiny to be processed. This was seen in 8 out of 16 samples ( $50 \%$ of samples )

4,2: Presence of extensive necrotic material which was difficult to freeze. This was seen in 8 out of 16 ( $50 \%$ of cases)

Tiny specimens were either nodal tissue sent for evaluation or a core needle biopsy from a primary tumor.

4.3: Time taken for frozen section analysis:

Time taken for evaluation was 50-70 minutes in $68 \%$ cases.The average time taken for evaluation was 62 minutes.

4.4: Frozen section deferral rate : $16 / 179$ samples were deferred for final histopathological analysis, giving a deferral rate of $8.9 \%$.

4.5: Concordance and discordant of frozen section with final histopathology:

Of the 163 samples which were technically evaluable and interpretable, 148 were concordant with paraffin sections giving a concordance rate of $90.79 \%$. 17/163 samples were discordant with paraffin sections giving a discordant rate of $10.42 \%$

\section{6:Analysis of sensitivity, specificity, false positive and false negative rates of frozen section:}

163 specimens were adequate for evaluation on frozen section. Frozen section was reported positive for malignancy in 51 samples and out of these 48 were true positive (positive on paraffin section also and 5 were false positive (negative on paraffin section) Frozen section was reported negative for malignancy in 112 samples and out of these, 100 were true negatives ( negative on paraffin section) and 12 were false negative (positive on paraffin section) 
Thus the sensitivity, specificity, positive predictive value and negative predictive value of frozen section were $80 \%, 95.2 \%, 90.56 \%$ and $89.2 \%$ respectively.

\section{7: Analysis of discordant cases :}

There were 5 false positive reports. Two were mucinous ovarian neoplasms, one parotid tumor, one sentinel node from head and neck cancer and a follicular neoplasm of thyroid which were falsely reported as positive due to frozen artefacts. There were 7 false negative cases of which 2 were breast cancers, 2 peritoneal deposits from ovarian cancer, a case of pancreatic head mass, one inguinal node from primary melanoma and a margin status for head and neck cancer. The reasons for false negativity were sampling error in extensively necrotic tumors and interpretation errors.

\section{8:Subset analysis for sensitivity and specificity by type of sample:}

The sensitivity and specificity of frozen section evaluation of nodes was high at $94.1 \%$ and $98.1 \%$. The concordance rate for nodal evaluation was $97.2 \%$. The sensitivity and specificity for evaluation of margin status by frozen section were $75 \%$ and $100 \%$ respectively. The Concordance rate for evaluation of margin was $94.4 \%$. The sensitivity and specificity for primary diagnosis of tumor by frozen section were lower at $70.3 \%$ and $925 \%$ respectively and its concordance rate was $80.76 \%$

\section{Discussion}

The concordance and discordance rates of frozen section described in the literature varies between 92 to $98 \%$ and 1 to $7 \%$ respectively.[ 1 - 5 ].The college of American Pathologists have reviewed over 90,000 frozen sections from 461 institutions and have showed a concordance rate of 98.52\%.[ 7 ]. Discordance rates up to $11 \%$ have been documented. [ 8 ]. However, the accuracy of frozen section varies with the site of biopsy, type of specimen and diagnosis [ 7 ]The results of the present study showed an overall concordance rate of $90.79 \%$ and an overall discordance rate of $10.42 \%$. On subset analysis, the concordance rate for evaluation of nodes, margins and for evaluation of the primary for diagnosis were $97.2 \%, 94.4 \%$ and $80.76 \%$ respectively.

\section{1:Analysis of discordant cases :}

A case of follicular adenoma thyroid, two cases of adnexal masses, of which one was a borderline mucinous tumor, a case of sentinel node from head and neck squamous cell carcinoma and a suspected neck node from mucoepidermoid carcinoma Parotid were false positive on frozen section.

Frozen section artifacts cause architectural distortion and alteration in the nuclear cytoplasmic ratio, which resulted in interpretation errors in the evaluation of nodes and adnexal masses.

Follicular neoplasms of thyroid and borderline ovarian tumors have inherent limitations to be diagnosed by frozen section . [ 9 - 12 ]

A case of suspected inguinal node metastasis from melanoma foot, mesenteric nodes from small bowel lymphoma, core needle biopsies from cancer breast [ 2 cases ], a core needle biopsy from pancreatic head mass , cancer ovary with peritoneal deposits [ 2 samples ] and a margin from head and neck squamous cell carcinoma were false negative on frozen section.

The most common reason for false negativity was sampling error [ 8/12 samples ]. In addition, there are inherent limitations in the diagnosis of lymphoma, melanoma and head of pancreas mass by frozen section. The diagnosis of melanoma and lymphoma require serial sectioning and use of ancillary studies and hence the diagnosis is deferred for histopathological examination.[ 13,14 ]. Regarding pancreatic masses, a number of studies have confirmed the inaccuracy of frozen section in differentiating between malignancy and chronic pancreatitis. [ $15,16,17]$

\section{2: Subset analysis and corrected concordance rates:}

On subset analysis, it was found that the concordance rate of frozen section in evaluating nodes was 97.2\% and margin status was $94.4 \%$. However, the concordance rate of frozen section for primary diagnosis of tumor was only $80.76 \%$. But, after excluding melanoma, lymphoma, follicular neoplasm of thyroid, borderline ovarian cancer and pancreatic mass, which have their own limitations for diagnosis by frozen section, the corrected concordance rate was $89.36 \%$.

The corrected overall concordance and discordant rates were $93.08 \%$ and $6.9 \%$ respectively.

\section{3:Deferral rate:}

In this study, 8.9\%( 16/179) of samples were deferred for histopathological analysis. 87.5\% [ 14/16 ] of the samples were deferred due to technical difficulty of performing a frozen section, either due to inadequacy in freezing or processing. $12.5 \%$ [ 2/16 ] samples were deferred due to interpretation difficulty due to frozen artifacts. 


\section{4: Comparison with other studies[ TABLE I]}

The concordance rate of this study was comparable to other studies as shown in the table. The discordance and deferral rates were higher when compared to other studies. This was mainly due to technical errors which is expected to decrease with increase in experience with frozen sections and with strict adherence to quality control.[ 17-24]

\section{5:Diagnostic accuracy by type of tissue processed [TABLE II]}

The concordance rate of nodal evaluation, margin assessment, sentinel nodes and tumor deposits were more than $91 \%$ and comparable to other studies. The concordance rate of evaluation of primary tumor was $84.6 \%$. This was due to the inclusion of certain pathologies which are difficult to evaluate by frozen section.

The negative aspect of frozen section in this study was the time taken for frozen section. The average time was 62 minutes, which was much higher than the accepted time of twenty minutes. This was due to logistic issues inherent to the public sector like, power cuts, availability of personel etc. However, the time to frozen analysis gradually decreased as frozen sections became part of departmental protocol.

Table I The concordance rate of this study compared to other studies:

\begin{tabular}{|l|l|l|l|l|}
\hline & Study & Concordance rate & Discordance rate & Deferral rate \\
\hline 1 & CAP program, 1990 & $96.5 \%$ & $3.5 \%$ & $3.9 \%$ \\
\hline 2 & CAP review, Zabro, 1991 & $98.3 \%$ & $1.7 \%$ & $4.2 \%$ \\
\hline 3 & Mayo Clinic study & $97.8 \%$ & $2.2 \%$ & - \\
\hline 4 & CAP review, Novis, 1996 & $98.1 \%$ & $1.8 \%$ & $4.6 \%$ \\
\hline 5 & Wen, China study, 1997 & $92.6 \%$ & $3.6 \%$ & $4.7 \%$ \\
\hline 6 & Pakistan study, 2008 & $97.08 \%$ & $2.92 \%$ & $3.93 \%$ \\
\hline 7 & IOSR JDMS, 2013 & $92 \%$ & $2 \%$ & $6 \%$ \\
\hline 8 & This study & $93.08 \%$ & $6.9 \%$ & $8.9 \%$ \\
\hline
\end{tabular}

Table II diagnostic accuracy by type of tissue processed:

\begin{tabular}{|l|l|l|l|l|l|l|}
\hline & Type & No. & Concordance rate & Inconclusive & $\begin{array}{l}\text { False } \\
\text { positive }\end{array}$ & $\begin{array}{l}\text { False } \\
\text { negative }\end{array}$ \\
\hline 1 & Nodes & 79 & $97.2 \%[70 / 72]$ & {$[8.8 \%] 7 / 79$} & $1.38 \%[1 / 72]$ & 0 \\
\hline 2 & Tumor & 58 & $84.6 \%[44 / 52]$ & {$[10.3 \%] 6 / 58$} & $5.7 \%[3 / 52]$ & $9.6 \%[5 / 52]$ \\
\hline 3 & Margin & 19 & $94.4 \%[17 / 18]$ & 0 & 0 & $1 / 18[5.5 \%]$ \\
\hline 4 & $\begin{array}{l}\text { Sentinel } \\
\text { node }\end{array}$ & 13 & $91.6 \%[11 / 12]$ & {$[7.6 \%] 1 / 13$} & $8.3 \%[1 / 12]$ & 0 \\
\hline 5 & $\begin{array}{l}\text { Tumor } \\
\text { deposits }\end{array}$ & 10 & $100 \%[8 / 8]$ & {$[20 \%] 2 / 10$} & 0 & 0 \\
\hline
\end{tabular}

\section{Conclusion}

Frozen section is reliable and accurate for intraoperative evaluation of nodes, margin status and for the primary diagnosis of most tumors. However, it has limited value in the evaluation of certain tumors like melanoma, follicular neoplasms of the thyroid, lymphomas, borderline ovarian tumors and pancreatic mass lesions Tumors with a large necrotic component and very small samples pose challenges in evaluation by frozen section due to high sampling errors and technical difficulty respectively.Frozen section evaluation is an accurate means of intraoperative diagnosis . It is feasible to run a reasonably accurate frozen section facility at the public sector and thus and its efficacy can be validated. Hence, we would like to suggest development of the facility in all public sectors were oncological work is pursued.

\section{References}

[1]. Winship et al. Frozen sections: an avaluation of 1810 cases. Surgery. 1959: 45: 462-466

[2]. Ackerman. Et al. The indications for and limitations of frozen section diagnosis. Br. J Surg. 1959: 46: 336-350

[3]. Holaday. Et al. Ten thousand consecutive frozen sections. Am J Clin Pathol. 1974: 61 : 769-777

[4]. Dankwa et al. Frozen section diagnosis : an audit. J Clin Pathol. 1985; 38: 1235-1240

[5]. Rogers et al. Accuracy of frozen section diagnosis in a teaching hospital. Arch pathol lab Med. 1987; 111: 514-517

[6]. Sawady et al. Acduracy of and reasons for frozen sections : a correlative and retrospective study. Hum Pathol: 1988; 19: 1019-1023

[7]. Gephardt GN, Zarbo RJ. International comparison of frozen section consultation. A college of American Pathologists Q-probe study of 90,538 cases in 461 institutions.

[8]. Dahlin et al. Seventy five years experience with frozen sections at the Mayo Clin Proc. 1980; 55: 721-723

[9]. Herbert chen et al, Follicular lesions of thyroid, does frozen section evaluation alter operative management . Ann Surg. July 1995

[10]. Peggy Geomini et al, Diagnostic accuracy of frozen section diagnosis of adnexal masses.: a meta analysis. Gynaecological oncology. 96 [ 2005 ) 1-9

[11]. Amita Maheswari et al. Accuracy of intraoperative frozen section in the diagnosis of ovarian neoplasms: Experience at a tertiary oncology centre. WJSO,2006; 4:12

[12]. Coffey et al. Intraoperative consultation in gynaecologic pathology. Arch Pathol lab med. 2005; 129: 1544-57

[13]. Pieter. J. Tanis. Frozen section investigation of sentinel node in malignant melanoma and breast cancer. Annals of Surgical oncology. 2008 [ 3 ); 222-236 
The Realisitic Value Of Frozen Section In Intraoperative Decision Making In Malignancies....

[14]. Alexander stojadinovic et al. Value of frozen section analysis of sentinel lymph nodes for primary cutaneous melanoma. Ann. Surg. 2002. Jan; 235 [1) 92-98

[15]. Harris et al. Pancreatic cancer, unreliability of frozen section in diagnosis. South med J . 1995; 78: 1053-56

[16]. Campanade et al . Reliability and sensitivity of frozen section pancreatic biopsy. Arch Surg 1985; 120:283-88

[17]. Witz et al. Intraoperative pancreatic biopsy- a diagnostic dilemma. J . Surg. Oncol. 1989; 42: 117-119

[18]. Peter. J. Howanitz et al, The accuracy of frozen section diagnosis in 34 hospitals. Arch Pathol lab med; Vol 114, April 1990

[19]. Ferreiro JA ${ }^{1}$, Myers JL, Bostwick DG.Accuracy of frozen section diagnosis in surgical pathology: review of a 1-year experience with 24,880 cases at Mayo Clinic Rochester,Mayo Clin Proc. 1995 Dec;70(12):1137-41.

[20]. Juang.Liang Chang et al. Diagnostic accuracy of frozen section in Surgical Pathology. A retrospective analysis of 1084 frozen sections; J . Med. Scien;133-142, 1992

[21]. RAFAEL DENADAI PIGOZZI DA SILVA et al Diagnostic accuracy of frozen section tests for surgicaldiseases; Rev. Col. Bras. Cir. 2011; 38(3): 149-154

[22]. Shrestha S et al; Comparative Study of Frozen SectionDiagnoses with histopathology; Postgraduate medical journal of NAMS;Volume 9 | Number 2 | July-Dec 2009

[23]. Dr.K.Chandramouleeswari et al; Frozen and paraffin sections - Comparative study highlighting the concordance and discordance rates in a tertiary care centre; IOSR Journal of Dental and Medical Sciences; Volume 12, Issue 5 (Nov.- Dec. 2013), PP 26-30

[24]. Zubair Ahmad, Muhammad Abrar Barakzai, Correlation of intra-operative frozen section consultationwith the final diagnosis at a referral center in Karachi,Pakistan;Indian journal of pat holog y a nd m i crobi ology-51 (4), october-dec e m b e r 2008 